\title{
¿SOCIEDADES ARTIFICIALES? \\ Una introducción a la simulación social
}

\author{
SANDRA GONZÁLEZ BAILÓN* \\ Universidad de Oxford. Universidad de Barcelona
}

PALABRAS CLAVE ADICIONALES

Sistemas multi-agente, Individualismo metodológico, Emergencia, Normas, Redes.

\section{ADDITIONAL KEYWORDS}

Multi-agent Systems, Methodological Individualism, Emergence, Norms, Networks.

RESUMEN . Estas líneas tienen un doble objetivo: introducir la simulación social como técnica de análisis y ofrecer una panorámica de sus usos y posibilidades. El artículo comienza con un par de ejemplos que ilustran la práctica de la simulación. Sigue con una exposición de los aspectos metodológicos que la definen para, después, señalar las ventajas (e inconvenientes) asociadas al uso de esta técnica de análisis. El propósito de la simulación social, como el de las ecuaciones estadísticas o el de los tipos ideales, es el de generar modelos de la realidad que permitan construir o validar teorías acerca de sus regularidades. Lo que hace de la simulación una estrategia analítica novedosa no son los propósitos, sino los medios: algoritmos de programación que contienen las reglas de actuación de unos agentes que interactúan entre ellos y con el entorno. Las ventajas de la simulación social no son sólo experimentales sino también, y sobre todo, teóricas: sus modelos permiten encontrar los mecanismos que modelos como los estadísticos son incapaces de proporcionar.

ABSTRACT. These lines have a twofold objective: to introduce social simulation as a technique of analysis, and to offer a view of its uses and possibilities. The article starts with a couple of examples aimed to illustrate the practice of simulation. The methodological aspects associated to it are then exposed to, finally, highlight the pros (and cons) of its use. The aim of social simulation is the same as that of statistical equations and ideal types: to generate models of the social reality that help us to build or validate theories about its regularities. What turns social simulation into an innovative analytical strategy is not its objectives but rather its means: programming algorithms that contain the behavioural rules of agents that interact among them and with their environment. The advantages of social simulation are not only experimental but also, and specially, theoretic: it allows the search of the mechanisms that statistical models cannot provide.

* Agradezco a David Casassas, Jordi Mundó, José Antonio Noguera y Daniel Raventós sus amables ( $\mathrm{y}$ valiosos) consejos tras la lectura de versiones anteriores de este artículo. También agradezco a un evaluador anónimo sus (igualmente valiosos) comentarios y sugerencias.

E-mail: sandra.gonzalezbailon@nuffield.ox.ac.uk

Revista Internacional de Sociología (RIS)

Tercera Época, No 39, Septiembre-Diciembre, 2004, pp. 199-222. 
RIS

REVISTa INTERnacional DE SOctologta

№ 39, SEPTIEMBRE-DICIEMBRE, 2004

SANDRA GONZÁLEZ BAILÓN

\section{UN PAR DE EJEMPLOS PARA EMPEZAR}

¿Por qué las acciones de los individuos generan a menudo consecuencias imprevistas? ¿Por qué las intenciones de cada cual engendran procesos no intencionados por nadie? Estas son las preguntas que Schelling se planteó hace ya más de tres décadas en el clásico Micromotives and Macrobehavior (Schelling, 1978). Schelling se centró en un hecho común a todas las sociedades: que lo que cada individuo hace afecta las acciones de los otros individuos; y que lo bien o lo mal que cada cual pueda conseguir sus objetivos depende de lo que otras personas estén persiguiendo. De esa acción y reacción emergen patrones de comportamiento que, pese a haber sido generados por individuos, nos cuentan muy poco de sus intenciones. Experimentamos los efectos de vivir enzarzados constantemente y, sin embargo, seguimos interpretando mal sus consecuencias: ante una ciudad segregada racialmente, la reacción espontánea es pensar que sus ciudadanos no son muy favorables a la diversidad y a la tolerancia. Es posible que en muchos casos sea así. La pregunta que se planteó Schelling fue, ¿lo es en todos?

Para responderla, y para dar cabida al problema de las consecuencias no intencionales de la acción, Schelling hizo uso de una estrategia explicativa ingeniosa: construyó una sociedad artificial y la pobló de individuos en busca de vecindario (Schelling, 1969; Schelling, 1971). Los individuos, miembros de dos grupos distintos, habitan en las celdas de un mundo bidimensional por el que pueden moverse en busca de un nuevo hogar. Cada individuo tiene un vecindario determinado por los individuos que habitan en las casillas adyacentes, las que corresponden a los cuatro puntos cardinales. Y ninguno de ellos busca vivir en grupos separados, sólo contar con algunos vecinos de su misma identidad. Sus exigencias pueden ser, por ejemplo, del $40 \%$; es decir, que ya se den por satisfechos si menos de la mitad de sus vecinos son de su mismo color. La ejecución del modelo muestra que, dado que esas preferencias las comparten todos, las dinámicas de sus interacciones acaban generando un mapa en el que, en efecto, se dibuja una segregación de barrios (figura 1a).

La conclusión fundamental del modelo de Schelling es que, aunque los agentes no quieren vivir en grupos separados, los barrios que forman con sus interacciones acaban segregándose. Ninguno de los agentes tiene un conocimiento global, ni puede anticipar las consecuencias de sus decisiones agregadas: se limitan a mirar a su alrededor, a ponderar si sus vecinos son lo suficientemente iguales a ellos mismos, a decidir si eso les satisface, y a quedarse o moverse en consecuencia. Curiosamente, cuando los individuos son demasiado exigentes, y buscan un vecindario exclusivamente de su mismo color, el modelo no alcanza un equilibrio: su exigencia les fuerza a estar en peregrinación constante (figura 1c). La simulación de Schelling no sólo exhibe la disparidad entre micromotivos y macrocomportamientos; también explica el por qué de esa discrepancia: las redes de interacción e interdependencia que vinculan a los agentes generan dinámicas no lineales que 
Figura 1.

Modelo de Segregación de Schelling (Wilensky, 1998)

(a) exigencia $=\mathbf{4 0} \%$

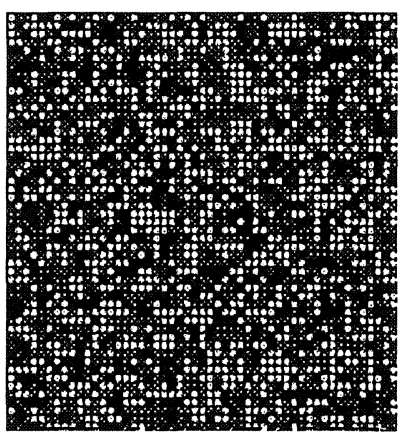

$\mathfrak{t}=0$

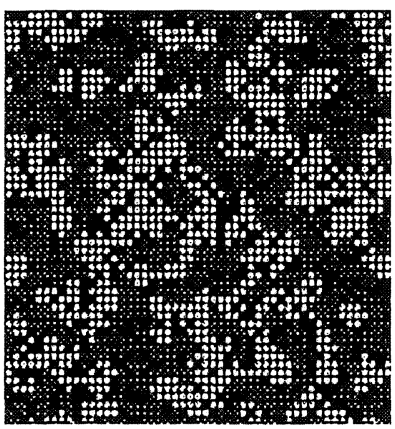

$\mathbf{t}=\mathbf{2 0}$

(b) exigencia $=\mathbf{2 0} \%$

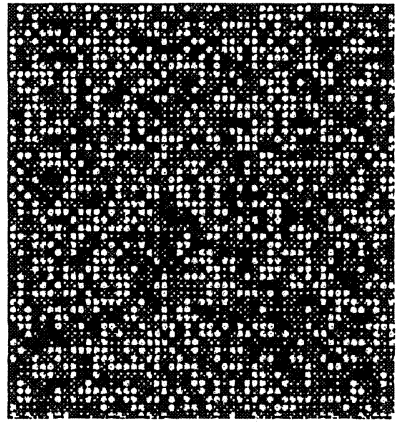

$t=0$

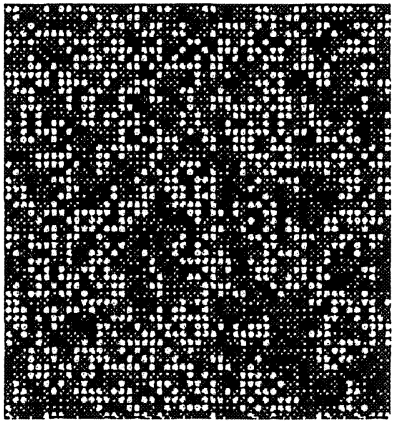

$t=20$

(c) exigencia $=90 \%$

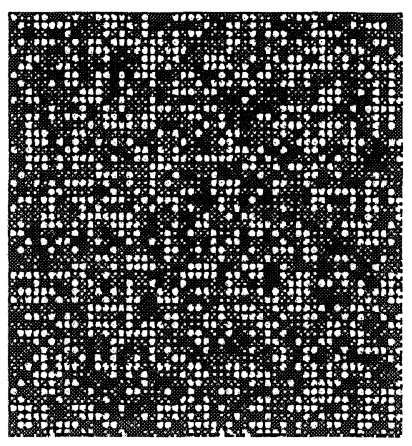

$t=0$

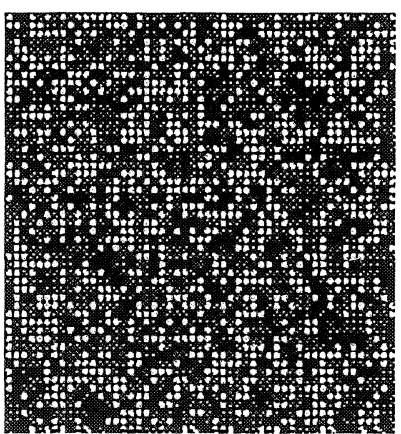

$t=20$ 
RIS

REVISTA INTERNACIONAL DE SOCIOLOGLA

№ 39, SEPTIEMBRE-DICIEMBRE, 2004

SANDRA GONZÁLEZ BAILÓN

acaban transformándose en esos patrones de segregación. Cuando un individuo decide dejar un barrio, su decisión no sólo le afecta a él, también afecta al resto del vecindario: su marcha puede haber truncado las expectativas de otros agentes que, en consecuencia, también deciden marcharse. Y de esa no linealidad surge lo inesperado, lo no previsto, las consecuencias no intencionadas de la acción.

$\mathrm{La}$ influencia de quienes nos rodean y con quienes entablamos nuestras interacciones es, pues, determinante. Una pregunta derivada de las intuiciones de Schelling es: si tendemos a dejarnos influir por quienes nos rodean, y todos los individuos estamos rodeados por alguien, $i$ cómo es posible que en procesos de diseminación cultural no se alcance la homogeneidad sino más bien lo contrario? Esta es la pregunta que se plantea Axelrod (Axelrod, 1997a). Y, para responderla, también él construye una sociedad artificial. Su objetivo fundamental es mostrar que la convergencia local no siempre deriva en una convergencia glebal. Los agentes de su modelo son, de nuevo, colocados en las casillas de un mundo bidimensional pero, esta vez, sin posibilidad de movimiento. La premisa de partida es que cuanto más similares son un actor y su vecino, más probable es que el actor adopte uno de los rasgos culturales del vecino. Esta premisa es, de hecho, la traducción de una intuición básica: la semejanza entre dos individuos promueve su interacción, y la interacción promueve una mayor semejanza. El reconocimiento de esta intuición hace aún más paradójica la pregunta de origen: si, a medida que interactúan, los individuos tienden a converger en sus creencias y actitudes, ¿por qué las diferencias no acaban desapareciendo? ¿Por qué los procesos de influencia social no construyen un mundo más homogéneo?

La respuesta a esta pregunta tiene que ver, de nuevo, con las consecuencias no intencionales y tos efectos colaterales de la acción. Si el individuo A decide interactuar con el individuo B porque comparten tres de cada seis opiniones, está abriendo la posibilidad de que, con la frecuencia de sus interacciones, ambos acaben convergiendo en una cuarta opinión. El individuo B, que, tras la convergencia, opina diferente en ese cuarto aspecto, ahora discrepa de un individuo $\mathrm{C}$ con el que, de no haberse dejado influir por A, hubiera coincidido. Su proximidad con A le aleja de $\mathrm{C}$ y eso genera consecuencias que no formaban parte de las intenciones de ninguno de ellos; en este caso, el comienzo de un proceso de polarización cultural. En el modelo, los rasgos culturales son representados por cadenas de números. El primero de ellos puede representar, por ejemplo, afiliación política; y puede tener diversos valores: 0 para la derecha, 1 para la izquierda. La decisión de interactuar de dos agentes depende de cuán similares son culturalmente: cuanto mayor es el porcentaje de rasgos con idéntico valor, mayor es la probabilidad de la interacción. Lo que la simulación muestra es que, a medida que los agentes convergen, los grupos se diferencian. Y lo hacen en función de cuatro parámetros: (i) el número de rasgos (que representan diversas dimensiones culturales); (ii) el número de valores para cada rasgo (un indicador de la diversidad cultural); (iii) el rango de interacción (el tamaño del vecindario); y (iv) el número de agentes (la población del sistema). 
La primera conclusión contraintuitiva es que, a medida que el número de rasgos culturales de los agentes aumenta, también aumenta la probabilidad de una convergencia cultural completa. Si bien en un principio parece razonable anticipar que cuantas más dimensiones culturales hay, más difícil es conseguir que todos converjan, el efecto resulta ser el contrario: al tener más rasgos que comparar, los agentes tienen más posibilidades de coincidir en al menos un rasgo; es decir, tienen más posibilidades de interactuar. Y con la interacción, viene la convergencia. Sin embargo, a medida que aumenta el número de valores por rasgo (a medida que aumenta la diversidad en cada una de las dimensiones culturales) el número de grupos culturalmente diferenciados crece. $\mathrm{Al}$ haber más valores para cada rasgo, existe una mayor probabilidad de que los agentes no tengan nada en común; es decir, disminuyen las posibilidades de interacción y, por lo tanto, de influencia social. Cuando el tamaño del vecindario se amplía para que la influencia no la ejerzan solamente los agentes más inmediatos sino también los de una segunda periferia, el número de regiones culturales disminuye. Algo que también sucede cuando el número de agentes (es decir, el tamaño de la población) aumenta.

¿Por qué son relevantes estos resultados? Según Axelrod, porque (i) permiten hacer una distinción teórica que, sin ellos, no se hubiera hecho, a saber: que hay dos aspectos culturales que evolucionan en direcciones opuestas; (ii) porque permiten poner a prueba un mecanismo de evolución cultural y evaluar el papel de las explicaciones funcionales: el hecho de que un rasgo desaparezca no siempre tiene que ver con que tenga menos ventajas o méritos; puede deberse, como muestra el modelo, al azar de las interacciones y a cuán vulnerable es una región cultural frente a eso; (iii) porque ilustran, de nuevo, la tesis de Schelling: que los macrocomportamientos a menudo nos cuentan muy poco de los micromotivos; en este caso, que la polarización cultural no tiene por qué ser el resultado de un proceso de evolución divergente sino, como en el modelo, de un mecanismo de convergencia con el vecino; y (iv) porque sugiere nuevas preguntas empíricas e hipótesis de trabajo.

Esta última ventaja es, por encima de todas las que destaca Axelrod, la que dota de mayor interés a la simulación social. Los experimentos de simulación no sólo permiten precisar las preguntas a un nivel que los experimentos mentales no alcanzan. También facilitan la validación y calibración empírica de los modelos (cuando se dispone de los datos para ello) y el diseño de estrategias de investigación para conseguir esos datos (cuando no existen o son insuficientes). El proceso de traducción de una teoría a algoritmos de programación es un filtro que atrapa hasta la más pequeña imprecisión conceptual. Y, a su vez, es un mecanismo de control de los elementos cuya fundamentación empírica está minada. En este caso, la simulación de procesos de influencia social hace evidente que son necesarios más detalles para poder anclar empíricamente las asunciones del modelo: ¿cuánta frecuencia de trato es necesaria para que un individuo se deje influir por otro? ¿Es la misma para todo el mundo? Si no lo es, ¿de qué depende? Y como 
RIS

REVISTA INTERNACIONAL DE SOCIOLOGIA

№ 39, SEPTIEMBRE-DICIEMBRE, 2004

SANDRA GONZÁLEZ BAILÓN

éstas, otras tantas preguntas que, bajo la retórica de la teoría, pasan muchas veces desapercibidas sin que por ello hayan recibido respuesta.

La construcción de un modelo de simulación permite, pues, detectar qué tipo de datos hacen falta para poder desglosar un problema hasta llegar a los bloques de construcción más básicos. Permite concretar el diseño de futuras investigaciones en el caso de que esos datos no estén disponibles. Y, además, permite explorar nuevas estrategias de intervención social: si hay algo que estos modelos demuestran es que más vale apostar por cambios en las condiciones de interacción local que emprender cambios globales centralizadamente. Éstos, para ser estables, siempre han de emerger de aquéllos. El modelo de Axelrod se aviene sólo con procesos de deriva cultural en los que no hay fuerzas institucionales que promuevan la adopción (o imposición) de determinadas opciones. Y se centra no tanto en los contenidos culturales (en su valor moral, ético o categórico) como en los mecanismos a través de los cuales estos contenidos se difunden y cristalizan en forma de grupos distintos. Pero el modelo puede ser modificado para trabajar con hipótesis alternativas y poner a prueba otros mecanismos. Esta flexibilidad es lo que dota a la simulación social de su mayor potencial explicativo.

\section{¿QUÉ ES, ENTONCES, LA SIMULACIÓN SOCIAL?}

Cuando Schelling ideó su modelo, no había ordenadores que pudieran exhibir en pantalla los resultados de la simulación. Sin embargo, su trabajo está considerado como uno de los clásicos en el área. Y hay una razón: el modelo reúne las características propias de los llamados sistemas multi-agente (SMA en adelante), los modelos de simulación que más posibilidades abren como marco de experimentación in silico. 'Simulación' puede significar, y ha significado, muchas cosas: desde las ecuaciones sobre la evolución de la economía global del Club de Roma, hasta las proyecciones demográficas, los autómata celulares, o los modelos de micro-simulación y de dinámica de sistemas (véase Gilbert and Troitzsch, 1999). Pero hay una serie de características que definen el modelo de Schelling, y que comparte el modelo de Axelrod, que hacen de los SMA unos modelos de simulación especialmente aptos para la investigación sociológica: su compromiso con el individualismo metodológico y con la prioridad de lo micro frente a lo macro; su interés por los sistemas masivamente locales y paralelos, carentes de autoridad central y generadores de procesos de mano invisible; la asunción de no racionalidad económica en sus agentes, aunque tampoco la excluya; y finalmente, la habilidad para capturar procesos emergentes que no pueden anticiparse intuitivamente, son las cuatro características que mejor sintetizan la naturaleza metodológica de esta estrategia de análisis.

La primera de esas características, el compromiso con el individualismo metodológico, tiene que ver con la propia lógica de construcción de los modelos. Los 
SMA son sistemas en los que la mayor parte de las tareas las ejecutan agentes que interactúan entre ellos y/o con el entorno (Epstein y Axtell, 1996: 4 y ss). Esos agentes disponen de una arquitectura en la que se especifican sus atributos y sus reglas de actuación. Los atributos (color y nivel de tolerancia en el modelo de Schelling; rasgos culturales, en el de Axelrod) se distribuyen heterogéneamente entre la población, y las reglas (moverse en busca de vecindario o mutar los rasgos culturales) se ejecutan en función de esas características individuales. Las estructuras sociales, o los comportamientos de los grupos, emergen de la interacción de esos agentes capaces de procesar únicamente información local. Una vez que han emergido, esas estructuras pueden tener un impacto en la acción individual. Pero, a la hora de definir y especificar el modelo, los bloques de construcción básicos siempre son los agentes y sus reglas de interacción.

La cuestión de hasta qué punto estas restricciones metodológicas son también restricciones teóricas ha recibido una atención especial en la literatura (vẻase Conte et al., 2001; también Hanneman, 1997). Sin embargo, éste es un debate con posturas irreconciliables a priori: que los sistemas sociales se deban analizar desde abajo (desde el comportamiento individual) o desde arriba (desde las instituciones que constriñen el comportamiento de los individuos) es algo que sólo puede responderse desde el matiz del problema a resolver, y no desde el modelo o la técnica. Pero dada la naturaleza de esta técnica en particular, es evidente que su uso prioriza la búsqueda de mecanismos, y que éstos, por definición, sólo se dan al nivel de los individuos (Hedström y Swedberg, 1998: 12). Sea como fuere, los términos en los que se ha llevado a cabo ese debate recuerdan al de la tensión acción-estructura que tantas páginas ha ocupado en la teoría sociológica (véase, por ejemplo, Alexander et al., 1987). Sin embargo, existe una diferencia: que la simulación permite llegar más lejos en su precisión conceptual. El esfuerzo integrador que hacen propuestas como la teoría de la estructuración de Giddens no deja de parecer ambiguo a la luz de los problemas que la construcción de un modelo de simulación ha de resolver. La simulación hace una lectura mucho más exigente de las teorías que afirman poder explicar procesos de estructuración y de emergencia. $Y$, en su exigencia, abre un potencial explicativo mucho mayor.

La segunda de las características de los SMA es que en estos sistemas la información se procesa de forma local y paralela; es decir, mediante la ejecución simultánea de unas reglas de actuación que rigen a un enjambre de agentes programados para procesar únicamente información local. Es precisamente este rasgo lo que hace de los SMA una herramienta idónea para el análisis de procesos complejos. La teoría de la complejidad tiene como objetivo estudiar el modo en que un gran número de actores (que pueden ser desde átomos hasta individuos) llevan a cabo sus interacciones y hacen emerger, con ellas, patrones de comportamiento globales de difícil predicción matemática (Axelrod, 1997b: 3). Mediante la simulación, diversos aspectos de esa complejidad pueden ser desglosados y analizados hasta desentrañar el tipo de impacto que ejercen en las dinámicas del 
RIS

REVISTA INTERnacional De SOcIologta

№ 39, SEPTIEMBRE-DICIEMBRE, 2004

sistema: por ejemplo, en qué sentido el nivel de tolerancia de los individuos es importante en la configuración de vecindarios segregados; o si hay algún otro factor, como la densidad de población, que también afecta a la evolución del sistema. El carácter descentralizado de los procesos que generan esas dinámicas (unos procesos que emprenden agentes que interactúan y se adaptan a su entorno local) es uno de los aspectos insitos a la complejidad. No hay una entidad única que procese la información centralizadamente, que esté al mando de la totalidad del sistema. Y sin embargo, el sistema funciona como si la hubiera.

El ejemplo canónico de esta propiedad es el de nuestra mente o, si se prefiere, el de esa percepción llamada 'identidad'. La noción de identidad es, en realidad, el engaño emergente de otro sistema masivamente paralelo: la red de conexiones neuronales que forman el tejido de nuestro cerebro (Hofstadter, 1992 (1979): 389 y ss). Esa imagen de nosotros mismos está en la raíz del sesgo que, ante la duda de por qué determinados sistemas se comportan como lo hacen, nos hace optar por el paradigma del "pensamiento centralizado" (Resnick, 1994: 3); es decir, por la búsqueda de causas que operen procesando globalmente la información, y que ordenen desde allí las acciones locales. Ante el mapa de una ciudad segregada, la tendencia es pensar que hay políticas de discriminación. Y, sin embargo, esa distribución puede deberse también a un mecanismo de retroalimentación positiva que refuerza y enquista los patrones derivados de unas reglas de interacción que no buscaban segregar (Resnick, 1994: 149). Los mecanismos de retroalimentación son una de las razones por las que los sistemas complejos pueden llegar a ser tan contraintuitivos. Y lo que hace que la simulación sea tan valiosa, cognitivamente, para abordar ese tipo de procesos.

La tercera característica, la racionalidad limitada de los agentes, es la que dota a los SMA de mayor ventaja, tanto teórica como empírica, frente a otras estrategias de análisis. Los agentes que pueblan las sociedades artificiales pueden ser heterogéneos en sus identidades, rasgos, preferencias, gustos o memoria; su razonamiento puede ser más o menos racional, inteligente o ingenuo; sus comportamientos pueden cambiar a lo largo del tiempo, bien porque aprendan de su experiencia pasada, bien porque aprendan de la experiencia de los demás; y pueden moverse por un entorno en busca de la satisfacción de sus preferencias, con mayor o menor miopía, para adaptarse a él o para modificarlo (Gaylord and D'Andria, 1998). El hecho de que estos agentes sean capaces de procesar únicamente información local es lo que les convierte en agentes adaptativos y lo que les aleja de los optimizadores racionales que asume el paradigma de la elección racional (Axelrod, 1997b: 4). Las reglas que rigen su comportamiento sólo pueden imponer demandas a la información de la que cada agente dispone y a su capacidad computacional (Epstein and Axtell, 1996: 4). Es decir, el tipo de demandas que se le pueden hacer a un individuo real en su ejercicio de racionalidad limitada (Simon, 1982). Los SMA hacen posible la construcción de modelos en los que los agentes ponen en práctica estrategias heurísticas para lidiar con un 
conocimiento imperfecto (Gilbert and Doran, 1994); y, por lo tanto, hacen posible la construcción de modelos de interacción más realistas.

Es esta flexibilidad en la estructura de los agentes la que marca una gran diferencia en las posibilidades que ofrecen los SMA frente a otras aproximaciones que, como la teoría de juegos, también formalizan situaciones de interacción social. En el año 1984, Axelrod publicó los resultados de un torneo basado en la iteración de diversas estrategias diseñadas para competir según los parámetros del Dilema del Prisionero (DP) (Axelrod, 1984). El objetivo era analizar las condiciones en las que las estrategias de cooperación emergen. Los resultados demuestran que la cooperación basada en la reciprocidad evoluciona y se mantiene incluso entre egoístas si la sombra del futuro (es decir, la probabilidad de interacciones futuras) es lo suficientemente larga. Pero esas conclusiones tienen un límite fundamental: los individuos disponen de información perfecta. En el año 1997, publica una compilación de artículos en los que, haciendo uso de SMA, revisa muchas de las asunciones de su trabajo anterior (Axelrod, 1997b). Una de las modificaciones es, precisamente, la de introducir "ruido" (perturbaciones) en la toma de decisión de los agentes, ya sea mediante el error en la percepción de lo que otros agentes hicieron en interacciones pasadas, ya mediante el error en la implementación de la estrategia escogida. Además, añade algo que la propia estructura del DP no permite hacer: analizar cómo emerge la cooperación cuando los agentes interactúan en grupos que vigilan y castigan a quien no coopera. Los SMA hacen del refuerzo de normas, y de los mecanismos de promoción (como la vigilancia, o la transmisión de rumores acerca de la reputación de algún agente), algo posible de modelar explícitamente. La arquitectura cognitiva que puede construirse en la programación de un agente es mucho más plástica y versátil que la que abre el recurso a preferencias y funciones de utilidad tal y como las formaliza la teoría de la elección racional.

Finalmente, el cuarto rasgo que caracteriza a los SMA es su habilidad para capturar procesos emergentes que no pueden anticiparse intuitivamente. Sin embargo, la noción de emergencia es ella misma muy intuitiva, difícil de definir y con un talón de Aquiles epistemológico: la duda de si llamamos emergencia a lo que, simplemente, no sabemos explicar (Kennedy y Eberhart, 2001: 18). La emergencia está asociada a los sistemas complejos, a las redes de interacción densas, y a la no linealidad que dificulta la predicción de la evolución de un sistema. Una forma de definirla es apelando al todo que es más que la suma de las partes (Johnson, 2001), a esa idea que, de hecho, está arraigada en el pensamiento social desde, como mínimo, los tiempos de la Ilustración Escocesa. Fue Ferguson quien, ya en 1767, dijo que la historia es el resultado de la acción de los individuos, pero no de su diseño intencional (Ferguson, 1996 (1767): sec. 3.2). Lo que ocurre con los SMA es algo muy parecido: el investigador define las reglas de actuación de los agentes, pero no define las reglas de evolución del sistema; define los términos de la interacción, pero no los resultados que han de emerger de ella. De ahí que se 
RIS

haya dicho que el potencial para la emergencia sea el mayor atractivo que ofrecen los SMA: el investigador define el mundo, se sienta y lo mira evolucionar (Page, 1998: xiii). La sorpresa inherente a toda investigación es especialmente saliente en la simulación social.

La mente ofrece, de nuevo, un símil interesante que permite abordar la definición de emergencia desde otra perspectiva. La filosofía de la mente ha ido virando en las últimas décadas hacia un consenso sobre el carácter superveniente de la mente, es decir, sobre el hecho de que ésta no puede reducirse a las propiedades físicas que le dan origen. El argumento se basa en la noción de "realizabilidad multiple", es decir, en el hecho de que un mismo estado mental (como el dolor) pueda ser realizado por estados físicos tan diversos que sea imposible establecer un vínculo causal de uno a otro (Fodor, 1975). Ante esto, algunos autores han dado un paso más para establecer un paralelismo con lo social: del mismo modo en que la mente no puede reducirse al soporte físico que le proporciona el cerebro, la sociedad no puede reducirse a los individuos, aunque proceda de ellos (Sawyer, $2001 ; 2002)$. Una misma institución social puede emerger de atributos individuales muy distintos y, en ese sentido, es irreducible a ellos, es decir, emergente.

A pesar de las dificultades inherentes a su definición, es posible sintetizar los elementos comunes a todo proceso emergente. En primer lugar, la emergencia siempre tiene lugar en sistemas formados por un gran número de componentes cuyo comportamiento sigue unas reglas muy simples. Esos componentes están interconectados por una red de interacciones que puede ir cambiando en el tiempo. Las interacciones no son lineales, de ahí que el comportamiento global no pueda obtenerse de la suma de las partes, ni anticiparse mediante el examen de las reglas que rigen el comportamiento de esas partes. Los procesos emergentes son patrones que persisten en el tiempo, aunque sus componentes vayan cambiando. Y, finalmente, son procesos que a menudo satisfacen sus propias leyes, distintas a las reglas que les dieron origen (Holland, 1998: 225-231). Estas características hacen que la simulación mediante SMA haya crecido en paralelo a los estudios sobre la emergencia: éstos no hubieran podido llevarse a cabo sin la existencia de aquélla. Su uso permite una acepción cada vez menos metafórica del todo que es más que la suma de las partes; y, con ello, una aproximación más analítica a la realidad social.

La simulación es, pues, una herramienta de análisis que, como tal, no contiene teoría. Pero permite construir buena teoría. Las teorías persiguen una tarea elemental: explicar el por qué de las regularidades que se observan en el mundo, y el por qué de sus excepciones. En ese empeño, el uso de la simulación contribuye a practicar los buenos hábitos: precisión a la hora de definir conceptos, coherencia lógica en su ensamblaje, especificación de leyes de comportamiento, detalle en la definición de las condiciones en las que esas leyes entran en juego, posibilidad de llevar a cabo una calibración guiada, y de validar los resultados con procesos empíricos concretos. Permite, ante todo, abordar de forma precisa preguntas muy 
bien definidas. La simulación mediante SMA permite hacer cualquier cosa que el investigador decida, con el único límite de su imaginación -y de la capacidad procesadora de las máquinas disponibles. La simulación social ofrece, en breve, el mayor potencial explicativo que ninguna otra técnica de experimentación de abasto social es capaz de proporcionar.

\section{VENTAJAS DE LA SIMULACIÓN}

Hay dos ventajas fundamentales asociadas al uso de la simulación social: la primera es que promueve una aproximación interdisciplinar a un mismo tipo de procesos; y la segunda es que, gracias a ello, permite salvar algunas de las insuficiencias asociadas al tipo de datos que tradicionalmente ha usado la investigación sociológica. La investigación basada en encuestas, la que más espacio ocupa en el quehacer de los sociólogos, cumple la función crucial de descubrir tendencias en la población; y los modelos estadísticos a ella asociados, la de detectar los factores que intervienen en esas tendencias. Pero ella misma no puede proporcionar la explicación de los fenómenos que detecta. Para que tal explicación pueda darse hay que mostrar cómo esas regularidades se crean y se mantienen a través de la acción e interacción de los individuos (Goldthorpe, 2000: 116). Y eso es algo que, a pesar de todo su potencial descriptivo, queda más allá de las posibilidades de la técnica estadística.

La teoría de la acción racional ha sido utilizada para suplir esa falta de mecanismos en, por ejemplo, estudios sobre la desigualdad en el sistema educativo (véase Boudon, 1974; también Goldthorpe, 2000: capítulo 9). Pero esa opción tiene más que ver con la parsimonia y el carácter formal de la teoría que con su realismo. Goldthorpe, por ejemplo, asume que los individuos son capaces de calcular el valor (o utilidad) del éxito y el fracaso escolar en función de la clase social de destino ( $y$, en consecuencia, de la de procedencia). No sólo asume que los individuos son capaces de obtener y procesar esa información con la misma habilidad sino que, además, toman sus decisiones aisladamente, más allá del poder de influencia del contexto o de sus redes de capital social. El argumento que suele esgrimirse en defensa de este tipo de estrategias es que la formalización tiene un coste: recortar el exceso de detalles. Pero el coste que la teoría de la elección racional tiene para este tipo de investigación sociológica es fundamental: básicamente, elimina el aspecto más social de la toma de decisión de los individuos. La simulación mediante SMA, en cambio, abre la posibilidad de ir más allá de las propiedades atomistas del agente racional sin detrimento de la formalización y la parsimonia.

Además de las exigencias cognitivas, pues, la teoría de la elección racional contiene en sus asunciones un problema añadido: el hecho de que no incorpore estructura en la interacción. Los modelos de interacción estratégica permiten que 
RIS

REVISTA INTERNACIONAL DE SOCIOLOGLA

NNo 39, SEPTIEMBRE-DICIEMBRE, 2004

SANDRA GONZÁLEZ BAILÓN

los agentes tengan en cuenta los intereses de los demás como parte de su cálculo de costes y beneficios. Pero asumen que todos tienen la misma posibilidad de interactuar con todos, lo cual es, de nuevo, una asunción poco realista. En la medida en que los SMA permiten modelar el entorno como un objeto con atributos y reglas de comportamiento propias, abren la posibilidad de añadir aspectos estructurales más allá de los premios y castigos que, a tal efecto, utiliza la teoría de juegos. En concreto, los SMA permiten incorporar redes de interacción en las que situar a los agentes; y esto permite, a su vez, ensanchar el rango de procesos sociales susceptibles de ser analizados en la búsqueda de mecanismos.

Las redes sociales han sido estudiadas sistemáticamente desde hace más de cinco décadas (Moreno, 1951). En ellas, los individuos son representados como nodos y sus relaciones, como aristas. La posición que los individuos ocupan en ese entramado de relaciones afecta a sus oportunidades y decisiones, de ahí que haya un paralelismo entre red y estructura social. La lista de asuntos que se han abordado haciendo uso del análisis de redes incluye temas tan variados como la movilidad social, la difusión y adopción de innovaciones, los procesos de decisión de élites, o la formación de coaliciones (para una lista más exhaustiva véase Wasserman et al., 1994: 5-6). Uno de los problemas fundamentales a los que se enfrenta el analista de redes es el de cómo acotar la recogida de datos, es decir, el de qué criterio utilizar para delimitar la red (Scott, 1991: 53-54). Los individuos no pueden interactuar arbitrariamente porque en su espacio de relaciones subyace una estructura. Esa estructura hace que unos individuos estén más conectados que otros, y que puedan utilizar su centralidad para tributar o favorecer a quienes, desde la periferia, sólo pueden acceder al resto de individuos indirectamente. Pero ni esa centralidad, ni su periferia son clases naturales, sino, más bien, conceptos que derivan del sistema de interacción particular que se está analizando y del criterio teórico que se sigue para recoger los datos. Milgram encontró, en 1967, una solución original al problema de cómo acotar la red (Milgram, 1967). En lugar de definir unos límites relevantes desde la teoría, ideó un experimento en el que estos límites emergían desde abajo, desde las decisiones de los individuos investigados.

El objetivo de Milgram era testar la hipótesis de los seis grados de separación, es decir, de hasta qué punto es cierto que, en el entramado de redes sociales, la distancia entre un par cualquiera de individuos se reduce a unos pocos grados de distancia, algo que viene conociéndose como el fenómeno Small World. En el experimento, cientos de personas fueron contactadas para que hicieran llegar un paquete postal a un destinatario desconocido. Sólo podían enviar el paquete a alguien con quien estuvieran conectados sin intermediarios y de quien supusieran que podía estar más cerca del objetivo. Lo que Milgram descubrió es que la mayoría de paquetes alcanzaban su objetivo tras haber pasado, como media, por las manos de sólo cinco o seis personas diferentes.

Lo que esos resultados indican no es que todo el mundo esté conectado con todo el mundo a través de únicamente seis pasos sino, más bien, que existe un 
reducido número de individuos que actúan como conectores. Ellos son los que sí están vinculados con todos las demás a través de unos pocos grados: la mayor parte de los paquetes que llegaron a su destino fueron entregados por una misma persona. Son esos individuos los responsables de que las redes sociales estén tan bien conectadas (Gladwell, 2001: 37). Aún así, la relevancia de los resultados del experimento de Milgram va más allá del número de 'saltos'necesarios para cruzar un país; 0 , para expresarlo en términos menos metafóricos, del número de aristas preciso para vincular dos nodos cualesquiera en una red. Lo que realmente importa de esos resultados es que desvelan una propiedad fundamental de los sistemas de interacción social, una estructura que les hace ser especialmente navegables. No importa en qué extremo se esté de la red, siempre es posible encontrar el otro extremo haciendo uso de los contactos, y de los contactos de esos contactos.

No obstante, una lectura cuidadosa de los resultados de Milgram revela que sus conclusiones están basadas en fundamentos empíricos más bien discutibles: ni el muestreo de los individuos que participaron en el experimento es suficientemente representativo, ni el porcentaje de cadenas de envío completadas (un 18\% del total de intentos) es significativo estadísticamente (Kleinfeld, 2002; Watts, 2003b: 132-135). Y sin embargo, a pesar de lo escuálido de la evidencia, y de que el experimento de Milgram jamás fue replicado para encontrar pruebas más convincentes, la idea de que el mundo es pequeño sigue más viva que nunca (Watts, 1999; Barabási, 2002; Buchanan, 2002; Watts, 2003b). El papel analítico que juega la simulación ha tenido un rol fundamental en ello: allí donde lo empírico no proporciona datos suficientes, los SMA han permitido construir un abanico de escenarios posibles en el que explorar las intuiciones de Milgram. Los modelos de simulación permiten crear unos agentes y unas reglas para establecer sus vínculos; y en ese ámbito es posible encontrar los distintos mecanismos que generan redes con propiedades Small World. El impacto que el fenómeno de los seis grados de separación tiene en la transmisión de información, de recursos o de cualquier otro aspecto de la interacción humana puede ser analizado sin tener que reducir los contenidos de esa transmisión a un paquete postal.

La razón que lleva a considerar el carácter interdisciplinar de la simulación social como una de sus virtudes se hace, aquí, evidente: el primer investigador en rescatar la importancia de las redes Small World lo hizo con el propósito de entender mediante qué mecanismos los grillos sincronizan sus cantos (Watts, 1999). Se le ocurrió que el patrón de interacciones que subyace a sus estrategias de apareamiento podía ser pensado como una red; y que cualquiera que fuera la estructura de esa red, tendría una influencia en el proceso de sincronización. De ahí pasó al estudio de redes de interacción humanas de naturaleza tan diversa como las que subyacen a la epidemia de enfermedades, al funcionamiento de los mercados, a la búsqueda de información $\mathrm{o}$ al organigrama de firmas que sobreviven a la crisis y al cambio (Watts, 2003b). Y se dio cuenta de que todas ellas tienen el mismo elemento en común que las redes de las que habían partido: son redes en 
RIS

REVISTA INTERNACIONAL DE SOCIOLOGLA

№ 39, SEPTIEMBRE-DICIEMBRE, 2004

SANDRA GONZÁLEZ BAILÓN

las que las cadenas de transmisión de un nodo cualquiera a cualquier otro no se extienden más de unos pocos pasos. Ahora, desde el departamento de sociología de la Universidad de Columbia, encabeza la investigación más ambiciosa que jamás se ha emprendido para saber si Milgram tenía razón y éste es, al fin y al cabo, un mundo pequeño (Watts, 2003a). Otros autores han virado en su interés por las redes informáticas hacia las redes sociales, validando la propiedad Small World en diversos sistemas de interacción humanos (Buchanan, 2002). Partiendo de intereses muy diversos, todos ellos han desembocado en el terreno sociológico. La razón estriba en su metodología: el uso de SMA no sólo les ha dotado de un lenguaje común, sino que, dadas las características de esta técnica de análisis, la realidad social emerge de repente como uno de sus objetivos naturales.

¿Qué papel desempeña, entonces, la simulación en la investigación sociológica? Uno doble: en primer lugar, permite hacer lo que procedimientos estándares no facilitan, como validar experimentos costosos, o emprender análisis que van más allá de lo puramente empírico. Determinar cuál de las redes Small World 'matemáticamente posibles' es más eficaz en la transmisión de información o recursos, y cuál de ellas se asemeja más a las redes sociales que operan en el mundo real, es un ejemplo de lo que la simulación social contribuye a hacer. Además, la simulación abre la vía a un trabajo interdisciplinar que no sólo aporta una técnica de análisis común sino también, y sobre todo, nuevos horizontes teóricos. Un ejemplo de ello es el que proporciona el análisis de las normas sociales.

El estudio de las condiciones en las que éstas emergen y se mantienen es uno de los más recurrentes en la literatura sociológica (Hechter and Opp, 2001; Hechter et al., 1990). Se han hecho esfuerzos por integrar su explicación al amparo de la teoría de la elección racional (Coleman, 1990; Ullmann Margalit, 1977) y como alternativa a ésta (Elster, 1989). Sin embargo, el carácter espontáneo y no intencional de las normas no acaba de encajar en estas aproximaciones, basadas exclusivamente en el paradigma de la acción y, por lo tanto, opuestas por definición al funcionalismo. El problema estriba en que la única forma de explicar el orden espontáneo, el diseño sin diseñador, es apelando a funciones (para una defensa del papel que juegan las explicaciones funcionales en las ciencias sociales véase van Parijs, 1981; véase también Dennett, 1995). Una explicación funcional de las normas sociales debería dar cuenta de los mecanismos que vinculan las consecuencias beneficiosas de su existencia (por ejemplo, escapar de un estado natural caótico) con su mantenimiento; es decir, necesita dar cuenta de un mecanismo de refuerzo. El papel que juegan los agentes, conscientes de las ventajas asociadas al respeto de las normas, es necesario, pero no suficiente, para explicar por qué de las múltiples normas posibles sólo se estabilizan unas; y por qué muchas de éstas son, a menudo, subóptimas para la satisfacción de sus intereses.

El estudio de las normas que, desde la inteligencia artificial, se hace a través de diversos SMA contribuye a esclarecer algunos aspectos cruciales. Hay estudios que demuestran que determinadas redes son especialmente eficaces en la 
emergencia de normas: dada la topología de algunos sistemas de interacción, las normas emergen más rápido y las convenciones se alcanzan antes (Delgado, 2002). Los SMA hacen posible establecer criterios de eficiencia y un vínculo de retroalimentación funcional que explique lo que el paradigma de la acción, por sí solo, no puede explicar. Los individuos encauzan sus interacciones en las posibilidades que les abre la red de relaciones que ocupan; y ésta, a su vez, evoluciona a la par que las interacciones. Si A no respeta una norma de reciprocidad que le une a $\mathrm{B}$, su vínculo se rompe y eso tiene un impacto en la estructura global de la red (en su topología). El sistema se encuentra en equilibrio cuando los agentes han encontrado una estrategia que satisface sus respectivas utilidades, pero también cuando la red ha alcanzado un diseño óptimo para la transmisión del tipo de información (o recursos) que forman parte de esas interacciones; por ejemplo, información acerca de la reputación de los agentes. Diversas estrategias cuajan en distintas redes porque éstas fomentan o frenan la proliferación de aquéllas. Es aquí donde entra en juego el mecanismo de retroalimentación que no puede capturar el paradigma de la acción.

Las redes son, pues, una pieza indispensable en el conjunto de mecanismos que hace que las normas emerjan y se mantengan. Mecanismos, no obstante, que no permiten explorar ni el análisis de redes que se ha venido practicando en la investigación sociológica (estático y con agentes inertes), ni la teoría de juegos (que asume que las interacciones se llevan a cabo en un mundo invertebrado). Hace ya tres décadas, Granovetter destacó la importancia que la estructura global de una red tiene en la evolución de los procesos que en ella se dan (Granovetter, 1973). Los SMA permiten llevar más lejos esa línea de investigación mediante la adición de dinámicas a la red y la habilitación de un entorno para la experimentación que previamente no existía.

Así pues, ¿cuáles son las ventajas de la simulación social? En primer lugar, que permite ir a donde otras estrategias de investigación no pueden llegar: propuestas como las de Milgram o Granovetter pueden convertirse en hipótesis de trabajo y someterse a una investigación más pormenorizada y exhaustiva. En segundo lugar, permite delimitar áreas donde hay un déficit de información empírica: apenas hay datos, por ejemplo, de redes completas y de las estrategias que siguen los individuos en esas redes para gestionar sus vínculos e interacciones. En tercer lugar, la simulación contribuye a diseñar propuestas de investigación que, en lugar de soslayarlas, se enfrenten a esas lagunas informativas: en el proceso de construcción de un modelo, surgen muchas de las preguntas que, de haber guiado la recogida de datos, hubieran hecho de ellos algo mucho más analítico y productivo. Finalmente, el uso de modelos de simulación social permite el enriquecimiento teórico asociado a todo trabajo interdisciplinar: el análisis de redes, o de normas sociales, que tanto tiempo lleva institucionalizado en la investigación sociológica, puede enriquecerse con el trabajo que, durante la última década, se ha venido desarrollando en ámbitos como el de la inteligencia artificial distri- 
buida. Aunque, a primera vista, parezcan líneas de trabajo muy remotas, en ellas se cuecen muchos de los intereses que han movido y mueven a la investigación sociológica. La contribución más importante que hace la simulación en este frente es la de facilitar el diálogo estableciendo un mismo lenguaje compartido.

\section{INCONVENIENTES DE LA SIMULACIÓN}

El uso de modelos de simulación social tiene dos inconvenientes fundamentales: el primero, de carácter técnico, tiene que ver con la necesidad de aprender un lenguaje de programación. La versatilidad que ofrecen los modelos de simulación se debe, en parte, a la libertad de movimiento que ofrece el no tener que trabajar con las asunciones implícitas de soportes lógicos preconstruidos. Pero aprender a programar conlleva unos costes de inmersión que, por formación profesional, pocos sociólogos están dispuestos a afrontar. La solución a este inconveniente pasa por hacer uso de la pericia técnica de quienes sí disponen de esa formación; es decir, pasa por la creación de grupos de trabajo interdisciplinares. El segundo inconveniente, de carácter empírico, es de mayor relevancia y tiene que ver con la disponibilidad de datos en los que anclar las asunciones del modelo. Cuando la simulación se usa para suplir la falta de datos, como en el estudio de redes de gran escala, su validación se hace, si no imposible, sí costosa. Y si el modelo no se valida, entonces no hay forma de evaluar su precisión. Sin un contraste empírico, los modelos de simulación no pueden desprenderse de un cierto aire de arbitrariedad que es, como mínimo, molesto.

Este inconveniente se deriva, en parte, del estatus metodológico de los SMA, que no se corresponden plenamente ni con los principios de la deducción, ni con los de la inducción: si bien empiezan con una serie de asunciones, su intención no es probar teoremas, sino generar datos con los que trabajar inductivamente; pero, al contrario de lo que ocurre con la inducción, esos datos no provienen del mundo real sino de un mundo simulado (sobre el estatus epistemológico de la simulación social véase Axelrod, 1997b: 3-4). Este estatus intermedio no sería problemático si la simulación no buscara suplir las deficiencias de otras estrategias de análisis en su búsqueda de mecanismos. Pero, para que los SMA puedan dar con explicaciones, es necesario que los datos que generan se correspondan con los patrones que se observan en el mundo real; y que, a partir de ahí, se pueda buscar en sus asunciones, rigurosamente especificadas en forma de reglas de comportamiento, los mecanismos que generaron esos patrones parejos a los de la realidad social. Es en esta empresa que la simulación se enfrenta a la mayor de sus dificultades.

Una de las razones estriba en que la mayor parte de la investigación sociológica se ha centrado en variables y en la búsqueda de correlaciones estadísticas. Como consecuencia, no es fácil encontrar datos que se avengan con el carácter eminentemente dinámico de los modelos de simulación (Chattoe, 2002).Además, dados 
los efectos de agregación, no es posible inferir comportamientos individuales de las tendencias globales; y la simulación se basa, fundamentalmente, en aquéllos. Es preciso, entonces, buscar ese tipo de datos en otras fuentes, que suelen provenir de la investigación cualitativa. Pero dada la poca coordinación entre las investigaciones de carácter cuantitativo (encargadas de analizar las tendencias) y las de carácter cualitativo (encargadas de profundizar en los casos concretos), construir una simulación en base a datos exclusivamente empíricos suele ser un suplicio. La habilidad de los modelos de simulación para detectar áreas donde se precisa más investigación es, sin duda, una de sus ventajas. Y existen las estrategias de análisis capaces de adquirir esos datos que no proporcionan las encuestas: por ejemplo, las entrevistas biográficas o las etnografias (Chattoe, 2002: 114 y ss). Pero mientras esos datos no se obtengan, la construcción de SMA se ve forzada a admitir que muchas de sus asunciones son, meramente, intuitivas.

Existen algunos modelos basados exclusivamente en datos empíricos. Uno de ellos formaliza un estudio de caso sobre cómo los gerentes de una compañía hidrológica gestionan situaciones de emergencia (Moss, 1998). El modelo permite explorar cuán eficaces son diversos modelos de organización para afrontar las incidencias que se dan en este sector; también qué estrategias de aprendizaje y colaboración entre los agentes que intervienen en su resolución demuestran ser más funcionales. Los resultados permiten evaluar el modo en que los gerentes reales se enfrentan a las crisis, y ponderar la relevancia de los criterios que siguen para determinar qué aspectos son más importantes en la toma de decisiones que ha de solucionar el problema. Es decir, los resultados de este modelo permiten valorar hasta qué punto las estrategias de gestión de incidentes reales son, o no, las más óptimas. Pero, al margen de esto, las conclusiones son de una naturaleza tan particular que apenas se pueden sacar conclusiones teóricas que vayan más allá del caso concreto.

Otros ejercicios de simulación ni siquiera intentan resolver el problema del anclaje empírico: en ellos, los modelos se convierten en un objeto de análisis per se, y los investigadores se limitan a determinar la importancia relativa que sus diversas asunciones tienen en los procesos y datos que el modelo genera. Un ejemplo de este tipo de estrategia lo proporciona el Sugarscape, uno de los SMA que mayor impacto ha tenido en el ámbito de la simulación social (Epstein y Axtell, 1996). En esta sociedad artificial los agentes se mueven sobre una red de celdas que producen recursos renovables. Los agentes pueden disponer de esos recursos bien para consumir o bien para almacenar y utilizarlos, posteriormente, como moneda de cambio. Los recursos están desigualmente repartidos y los agentes se mueven en su búsqueda intentando maximizar la cantidad obtenida. Dado que estos agentes tienen capacidades y metabolismos distintos, unos son más hábiles que otros para sobrevivir y prosperar adaptándose al entorno. Introduciendo rasgos culturales o cambios climáticos, el modelo reconstruye una protohistoria en la que surgen tribus, se dan migraciones, colisiones y enfrentamientos, intercambios eco- 
nómicos, asimilación cultural y otros tantos fenómenos que se generan partiendo, exclusivamente, de la interacción local. Pero, a pesar del interés conceptual que, sin duda, el Sugarscape ofrece, lo cierto es que frente a este tipo de modelos es difícil evitar la pregunta de qué hacer con ellos.

Un problema añadido al de la validación es el de la escasa replicación de los modelos para ver si sus resultados son consistentes. Como sucede con los resultados de cualquier otro experimento, es necesario verificar que los resultados de la simulación se deben a causas substantivas y no a meros accidentes derivados de, por ejemplo, errores de programación. La forma de llevar a cabo esta evaluación consiste en reimplementar los SMA en un entorno distinto, haciendo uso de otras plataformas o de otros lenguajes de programación. Existen algunos ejemplos que ilustran los procedimientos y resultados que se derivan de esta comparación sistemática, algo que se ha venido en llamar "alienación" de modelos (Axelrod, 1997b: 181 y ss). Estos ejemplos demuestran que, a menudo, unos modelos pueden subsumirse en otros y alcanzar, con ello, una mayor generalidad en los resultados. Pero lo común es que cada investigador haga de su modelo un empeño individual, con asunciones que parten de cero, y que dificultan que la investigación con SMA se integre en una empresa colectiva capaz de consolidar una acumulación del conocimiento.

La riqueza de detalles y la heterogeneidad inherente a la arquitectura de los SMA exige una riqueza en el detalle empírico con la que poder justificar las elecciones hechas en la construcción del modelo. Es necesaria información acerca de los procesos de decisión, de cómo los agentes recogen la información, de cómo la procesan, de las diferentes condiciones bajo las que sus interacciones tienen lugar, $\mathrm{y}$ de las consecuencias que generan escenarios cambiantes. También es necesario determinar cómo los agentes usan su historia pasada para aprender y mejorar sus estrategias, cómo el vecindario ejerce su impacto, cuáles son los umbrales de influencia, y cuál es la estructura de las interacciones. Es necesario, en breve, el tipo de información que las encuestas no pueden proporcionar, centradas como están en los resultados agregados y en creencias y valores medidos fuera de los contextos de interacción. Las consecuencias son, en el peor de los casos, que los modelos de simulación se convierten en juguetes abstractos que escenifican mundos posibles que apenas tienen que ver con la realidad; $y$, en el mejor, que es difícil validar los modelos $\mathrm{y}$, por tanto, construir representaciones fiables de los procesos analizados.

Lo que este problema identifica es la necesidad de buscar formas alternativas de conseguir datos sobre los que construir modelos sólidos. Hay varias opciones: integrar la investigación cuantitativa y cualitativa; hacer uso de las ciencias cognitivas para fundar la arquitectura de los agentes; o encontrar objetos de estudio de los que se disponga de una descripción empírica muy detallada. Pero las deficiencias en la disponibilidad de datos hace que la tarea de anclar los modelos pueda convertirse en un ejercicio ad hoc que desgaje aún más los procedimientos 
usados por esta técnica. La simulación también puede ser usada para crear datos de los que no se dispone, como cuando se cuenta con suficiente información sobre las acciones de los individuos pero no sobre sus consecuencias. Entonces el problema no concierne a la recogida de datos, sino a la validación. En estas circunstancias, que el modelo sea más o menos arbitrario depende de cuán precisas son las teorías en las que se basa. Y eso es algo que ya no depende de la técnica. La simulación social no deja de ser un mero instrumento.

El objetivo de la simulación social es, pues, analizar al mismo tiempo las dinámicas de las acciones individuales y las dinámicas de los resultados colectivos. Pero este potencial explicativo, que es su mayor ventaja, es también su mayor inconveniente. Es una ventaja porque permite buscar los mecanismos que la investigación basada en encuestas no permite buscar: los SMA están basados en las interacciones de los individuos y en cómo estas interacciones se agregan para formar propiedades emergentes, unas propiedades que podemos detectar y describir haciendo uso del análisis estadístico, pero que éste no permite explicar en un sentido causal fuerte. La simulación construye sistemas que, como los sociales, presentan una distribución masivamente local y paralela de las acciones individuales. Es esta característica la que permite testar diversas asunciones sobre los microfundamentos que subyacen a los procesos sociales. Pero éste es también su principal inconveniente porque no es fácil encontrar los datos que le den relevancia empírica. Por eso, las replicaciones son poco comunes: dado que las asunciones vienen dictadas por las intuiciones de los autores más que de evidencias concretas, no hay líneas de trabajo sistemáticas en las que converjan distintas investigaciones. Por su parte, la falta de teorías suficientemente concretas no contribuye a mejorar los problemas derivados de las lagunas que abre la evidencia empírica. Pero, finalmente, tanto las lagunas empíricas como las teóricas, son algo que no responden a un fallo intrínseco de esta técnica de análisis, sino que, más bien, la inhabilitan. Es evidente que esto perjudica a su implementación. Pero también lo es que la simulación, como técnica, no tiene por qué responsabilizarse de ello.

\section{CONCLUSIÓN}

La respuesta a la pregunta de por qué es importante la simulación para la investigación sociológica depende del tipo de sociología que se quiera practicar. En palabras de Boudon existen cuatro alternativas: la sociología de cámara o informativa, encargada de producir datos orientados al diseño y evaluación de políticas públicas; la sociología crítica o comprometida, que identifica los defectos de la sociedad y propone remedios para subsanarlos; la sociología expresiva o estética, cuyo propósito es describir los fenómenos sociales a partir del significado que le dan los protagonistas; $y$, finalmente, la sociología cognitiva o científica, cuyo objetivo fundamental es identificar y resolver fenómenos sociales que resultan 
enigmáticos (Boudon, 2002). Los modelos de simulación contribuyen a practicar este último tipo de sociología. Y lo hacen mediante la especificación de las reglas de comportamiento local que han de generar los procesos agregados con los que normalmente trabaja la investigación social. Es desde esos comportamientos agregados que los enigmas pueden detectarse. Pero su resolución depende de la identificación de los mecanismos que les subyacen.

El artículo se ha abierto con dos ejemplos que ilustran el tipo de problemas que la construcción de sociedades artificiales, y la experimentación que éstas posibilitan, contribuyen a resolver. Ha proseguido con una enumeración de los principios metodológicos asociados al uso de esa estrategia analítica: su compromiso con el individualismo metodológico, su interés por los sistemas masivamente locales y paralelos, la racionalidad limitada de los agentes y la habilidad para capturar procesos emergentes son los elementos que mejor caracterizan la naturaleza metodológica de la simulación social. De entre las ventajas se ha destacado el carácter interdisciplinar de sus modelos, y las aportaciones teóricas que pueden devenir de ello. El estudio de las condiciones en las que emergen y se mantienen las normas sociales ofrece un ejemplo del tipo de contribución que puede hacer el uso de modelos de simulación social. Entre los inconvenientes, destaca la escasa disponibilidad de datos empíricos en los que anclar las asunciones, tan pormenorizadas, de los modelos. Ni las bases de datos recabadas con encuestas, ni las teorías esbozadas sobre las regularidades que en ellas se encuentran están diseñadas para encajar en las exigencias de la simulación social. Pero en la medida en que ésta fuerza a repensar las teorías para hacerlas más analíticas e informativas, la no disponibilidad de datos está destinada a dejar de ser un problema y pasar a ser uno de los puntos a resolver en la agenda. Dada la flexibilidad de esta estrategia de análisis para resolver enigmas y para encontrar los mecanismos que no pueden encontrase haciendo uso de otras estrategias más convencionales, la simulación está llamada a ser una de las herramientas fundamentales en el quehacer sociológico de los próximos años.

\begin{abstract}
ANEXO
Este anexo tiene como finalidad poner a disposición del lector una serie de recursos electrónicos de interés para la simulación social. El listado incluye lenguajes de programación, listas de distribución, revistas especializadas y páginas desde las que se puede obtener información adicional sobre varios centros de investigación, cursos y conferencias. La relación no pretende ser exhaustiva y tiene como único objetivo abrir vía a quienes deseen profundizar en el uso de esta técnica de análisis.
\end{abstract}


Herramientas de programación

Centros de investigación

Lista de Distribución

Asociaciones

Revistas

Talleres
NetLogo

http://ccl.northwestern.edu/netlogo/

RePast

http://repast.sourceforge.net/index.html

Swarm

http://wiki.swarm.org/wiki/Main_Page

Center for Research in Social Simulation (CRESS)

http://cress.soc.surrey.ac.uk

Center for Computational Analysis of Social and Organizational Systems (CASOS)

http://www.casos.cs.cmu.edu

Complexity in Social Science http://www.irit.fr/COSI/

http://www.jiscmail.ac.uk/lists/simsoc.html

The European Social Simulation Association (ESSA) http://www.essa.eu.org/

North American Computational Social and Organization Science (NAACSOS)

http://www.casos.cs.cmu.edu/naacsos/index.php

Pacific Asian Association for Agent-based Approach in Economic \& Social Complex Systems (PAAA) http://www.paaa.econ.kyoto-u.ac.jp/

Journal of Artificial Societies and Social Simulation http://jasss.soc.surrey.ac.uk/JASSS.html

International Journal in Computer Simulation http://www.cs.umr.edu/ijcs/

ZUMA Simulation Workshop http://www.uni-koblenz.de/FB4/Institutes/IWVI /AGTroitzsch/Teaching/current_teaching/ZUMA

ZUMAAdvanced Simulation Workshop http://www.uni-koblenz.de/FB4/Institutes/IWVI /AGTroitzsch/Teaching/current_teaching/ZUMA-ASW 
R IS

REVISTA INTERNACIONAL DE SOCIOLOGIA

\section{REFERENCIAS BIBLIOGRÁFICAS}

ALEXANDER, J.C., B. GIESEN, R. MÜNCH y N.J. SMELSER (1987), The Micro-Macro Link, Berkeley, University of California Press.

AXELROD, R. (1984), The Evolution of Cooperation, Nueva York, Basic Books [La evolución de la cooperación, Madrid, Alianza, 1986].

(1997a), "The Dissemination of Culture. A model with local convergence and global polarization", Journal of Conflict Resolution, vol. 41, pp. 203-226.

(1997b), The Complexity of Cooperation. Agent-based Models of Competition and Collaboration, Princeton, Princeton University Press.

BARABÁSI, A. L. (2002), Linked. The New Science of Networks, Cambridge, Perseus.

BLOKLAND, T. (2003), Urban Bonds. Social Relationships in an Inner City Neighbourhood, Cambridge, Polity.

BOUDON, R. (1974), Education, Opportunity and Social Inequality, New York, Wiley.

(2002), "Sociology that Really Matters", European Sociological Review, vol. 18, pp. 371-378.

BUCHANAN, M. (2002), Nexus: small worlds and the groundbreaking science of networks, Nueva York, W.W. Norton.

CHATTOE, E. (2002), "Building Empirically Plausible Multi-Agent Systems: a Case Study of Innovation Diffusion", en B. Kerstin, D. Cañamero y B. Edmonds, Socially Intelligent Agents: Creating Relationships with Computers and Robots, Multi-Agent Systems, Artificial Societies and Simulated Organisations, Dordrecht, Kluwer.

COLEMAN, J. S. (1990), Foundations of Social Theory, Cambridge, Harvard University Press.

CONTE, R., B. EDMONDS, S. MOSS y R.K. SAWYER (2001), "Sociology and Social Theory in Agent Based Social Simulation: a Symposium", Computational and Mathematical Organization Theory, vol. 7, pp. 183-205.

DELGADO, J. (2002), "Emergence of Social Conventions in Complex Networks", Artificial Intelligence, vol. 141, pp. 171-185.

DENNETT, D. (1995), Darwin's dangerous idea: evolution and the meanings of life, London, Allen Lane the Penguin Press [La peligrosa idea de Darwin: evolución y significado de la vida, Barcelona, Galaxia, Gutenberg, 1999].

DIETZ, R.D. (2002), "The estimation of neighborhood effects in the social sciences: an interdisciplinary approach", Social Science Research, vol. 31, pp. 539-575.

ELSTER, J. (1989), The Cement of Society, Cambridge, CUP [El cemento de la sociedad, Barcelona, Gedisa, 1991]. 
EPSTEIN, J. M. y R.AXTELL (1996), Growing Artificial Societies. Social Science from the Bottom Up, Cambridge, MIT Press.

FERGUSON, A. (1996 [1767]), An Essay on the History of Civil Society, Cambridge, Cambridge University Press.

FODOR, J. (1975), The Language of Thought, Nueva York, Thomas Crowell.

GAYLORD, R.J. y L. L.J. D'Andria (1998), Simulating Society. A Mathematica Toolkit for Modeling Socioeconomic Behavior, Nueva York, Springer-Verlag.

GILBERT, N. y J. DORAN (1994), Simulating Societies. The computer simulation of social phenomena, London, UCL Press.

GILBERT, N. y K. G. TROITZSCH (1999), Simulation for the Social Scientist, Buckingham, OUP.

GLADWELL, M. (2001), The Tipping Point. How little things can make a big difference, London, Abacus.

GOLDTHORPE, J. H. (2000), On Sociology. Numbers, narratives, and the integration of research and theory, Oxford, Oxford University Press.

GRANOVETTER, M. (1973), “The strength of weak ties", American Journal of Sociology, vol. 78 , pp. $1360-1380$

HANNEMAN, R. y S. PATRICK (1997), "On the Uses of Computer-Assisted Simulation Modeling in the Social Sciences", Sociological Research Online, vol. 2, pp. http://www.socresonline.org.uk/ socresonline $/ 2 / 2 / 5 . \mathrm{html}$.

HECHTER, M. y K.D. OPP (ed.) (2001), Social Norms, Nueva York, Russell Sage Foundation.

HECHTER, M., K.D. OPP, y R. WIPPLER (eds.) (1990), Social Institutions: their emergence, maintenance, and effects, Nueva York, Walter de Gruyter.

HEDSTRÖM, P. y K. SWEDBERG (eds) (1998), Social Mechanisms. An analytical approach to social theory, Cambridge, Cambridge University Press.

HOFSTADTER, D.R. (1992 [1979]), Gödel, Escher, Bach. Un eterno y grácil bucle, Barcelona, Tusquets.

HOLLAND, J. H. (1998), Emergence. From chaos to order, Oxford, Oxford University Press.

JOHNSON, S. (2001), Emergence. The connected lives of ants, brains, cities, and software, Nueva York, Scribner.

KENNEDY, J. y R. EBERHART (2001), Swarm Intelligence, San Francisco, Morgan Kaufmann.

KLEINFELD, J. (2002), "The Small World Problem”, Society, vol. 39, pp. 61-66, http://www.uaf.edu/ northern/big_world.html.

MILGRAM, S. (1967), "The Small World problem", Psychology Today, vol. 2, pp. 60-67. 
MORENO, J.L. (1951), Sociometry, experimental method and the science of society, Nueva York, Beacon House.

MOSS, S. (1998), "Critical Incident Management: an Empirically Derived Computational Model", Journal of Artificial Societies and Social Simulation, vol. 1, http://www.soc.surrey.ac.uk/ JASSS/1/4/1.html.

PAGE, S.E. (1998), “Foreword”, en R.J. Gaylord y L.J. D'andria (eds), Simulating Society. A Mathematica Toolkit for Modeling Socioeconomic Behaviour, Nueva York, Springer-Verlag.

PARIJS, P. V. (1981), Evolutionary Explanation in the Social Sciences: an emerging paradigm, London, Tavistock.

RESNICK, M. (1994), Turtles, Termites, and Traffic Jams. Explorations in Massively Parallel Microworlds, Cambridge, The MIT Press.

SAWYER, R.K. (2001), "Emergence in Sociology: Contemporary Philosophy of Mind and Some Implications for Sociological Theory", American Journal of Sociology, vol. 107, pp. 551-585.

(2002), "Durkheim's Dilemma: Toward a Sociology of Emergence", Sociological Theory, vol. 20 , pp. 227-247.

SCHELLING, T.C. (1969), "Models of Segregation", The American Economic Review, vol. LIX, pp. 488-493.

(1971), “Dynamic Models of Segregation”, Journal of Mathematical Sociology, vol., pp. 143-186.

(1978), Micromotives and Macrobehavior, London, Norton [Micromotivos y macroconducta, México, Fondo de cultura Económica, 1989].

SCOTT, J.P. (1991), Social Network Analysis: a Handbook, London, Sage.

SIMON, H.A. (1982), Models of Bounded Rationality, Cambridge, MIT Press.

ULLMANN MARGALIT, E. (1977), The Emergence of Norms, Oxford, Oxford University Press.

WASSERMAN, S., K. FAUST y D. ACOBUCCI (1994), Social Network Analysis: Methods and Applications, Cambridge, Cambridge University Press.

WATTS, D.J. (1999), Small Worlds. The Dynamics of Networks between Order and Randomness, New Jersey, Princeton University Press.

(2003a), http://smallworld.columbia.edu.

(2003b), Six Degrees. The Science of a Connected Age, Londres, William Heinemann.

WILENSKY, U. (1998), "NetLogo Segregation model", Center for Connected Learning and Computer-Based Modeling, Evanston, Northwestern University.

http://ccl.northwestern.edu/netlogo/models/Segregation. 